\title{
Carcinoma of the cervical stump: Fifty years of experience
}

\author{
ANN-CATHRIN HELLSTRÖM ${ }^{1}$, KRISTINA HELLMAN ${ }^{1}$, B. FOLKE PETTERSSON ${ }^{1}$ and SONIA ANDERSSON ${ }^{2}$ \\ ${ }^{1}$ Gynecological Oncology Radiumhemmet, Department of Oncology, Karolinska University Hospital, Solna; \\ ${ }^{2}$ Department of Women and Child Health, Division of Obstetrics and Gynecology, Karolinska \\ University Hospital, Solna, Karolinska Institutet, 17177 Stockholm, Sweden
}

Received December 16, 2010; Accepted January 13, 2011

DOI: $10.3892 /$ or.2011.1228

\begin{abstract}
A series of patients with carcinoma of the cervical stump in relation to age, clinical stage, histopathology, changes in relative incidence, treatment outcome and longterm survival, were studied and the findings were compared with matched controls that have an intact uterus and cancer of the cervix. Of 8,028 women treated for invasive cervical carcinoma between 1959-2004, 161 were diagnosed with stump cancer, accounting for $2.0 \%$ of all cervical cancers. The mean time interval between subtotal hysterectomy and stump cancer diagnosis was 17.6 years, with a range of 1-46 years. In $80 \%$ of cases, symptoms drove the patient to seek medical attention and postcoital, intermenstrual or postmenopausal bleeding was the main reason. Among 161 stump cancer cases $89 \%$ were squamous cell carcinoma (SCC) and the remaining 17 cases were adenocarcinomas (AC). Cumulative cause-specific survival rate was significantly worse for adenocarcinoma than for squamous cell carcinoma (SCC) of the cervical stump (Log-rank $\mathrm{p}=0.027$, Cox-Mantel $\mathrm{p}=0.015$, Cox F-test $\mathrm{p}=0.01)$. The stump cancer cases show a worse stage profile compared with the cancer cases in intact uterus. We conclude that the total effect of stump cancers following subtotal hysterectomies is not to be neglected.
\end{abstract}

\section{Introduction}

Total hysterectomies have routinely been performed in Sweden, even for benign gynecologic conditions and a number of women in our hospital region have undergone subtotal hysterectomies for more or less well-established reasons. A number of stump cancers have occurred, mostly among older women. Recently, the trend has been towards preserving the cervix by performing abdominal, laparoscopic, or vaginal subtotal hysterectomies. In the future, we may

Correspondence to: Dr Sonia Andersson, Department of Women and Child Health, Division of Obstetrics and Gynecology, Karolinska University Hospital, Solna, Karolinska Institutet, 17177 Stockholm, Sweden

E-mail: sonia.andersson@karolinska.se

Key words: cervical stump carcinoma, subtotal abdominal hysterectomy, prognosis therefore expect to see an increase in the number of cervical stump cancers.

The aim of this study is to describe a series of patients with carcinoma of the cervical stump. The study takes into account patient age, clinical stage, histopathology, changes in relative incidence, treatment outcome, and long-term survival, and compares the findings with matched controls having an intact uterus and cervical cancer.

\section{Materials and methods}

In the early 1900s treatment of cervical cancer (CC) in Sweden was centralized at Radiumhemmet in Stockholm. Initially patients were referred from all over Sweden, but treatment centers were gradually established in other parts of Sweden. In more recent years patients have been referred to Radiumhemmet from hospitals within the Stockholm-Gotland region, with a population of 1.8 million. The Stockholm method of CC treatment has become one of the leading methods in the world. In order to obtain reliable data of treatment results, a group of pioneering doctors including Professor Heyman (1917-1947) and Professor Kottmeier (1948-1971), established the first database of patients with $\mathrm{CC}$. These data included information on tumor stage, histopathology, treatment and follow-up results.

Out of 8.028 women treated for CC 170 women treated for invasive cervical stump carcinoma between 1959 and 2004, 9 had to be omitted; 3 cases were coincident cancers (1 case of $\mathrm{CC}$ and 2 of precancerous stages detected within one year of hysterectomy), 1 case had no histopathological verification, and 5 cases involved patients from foreign countries and could not be followed up. The material was reduced to 161 women with cervical stump carcinoma treated between 1959 and 2004, representing $2.0 \%$ of all treated CC.

Statistical analysis. For each case, 3 control cases were selected from the cohort of $\mathrm{CC}$ cases and matched according to year of treatment, stage, histology, and age ( \pm 5 years). Control cases were selected to correspond as closely as possible with the date of diagnosis of the index case. Due to the limited total cohort of treated cases, it was impossible to find three controls for every case of stump cancer, particularly for cases of adenocarcinoma in older age groups and for advanced stages (see tables presenting distribution of stages and controls by age, stage, and histopathology). Actuarial 


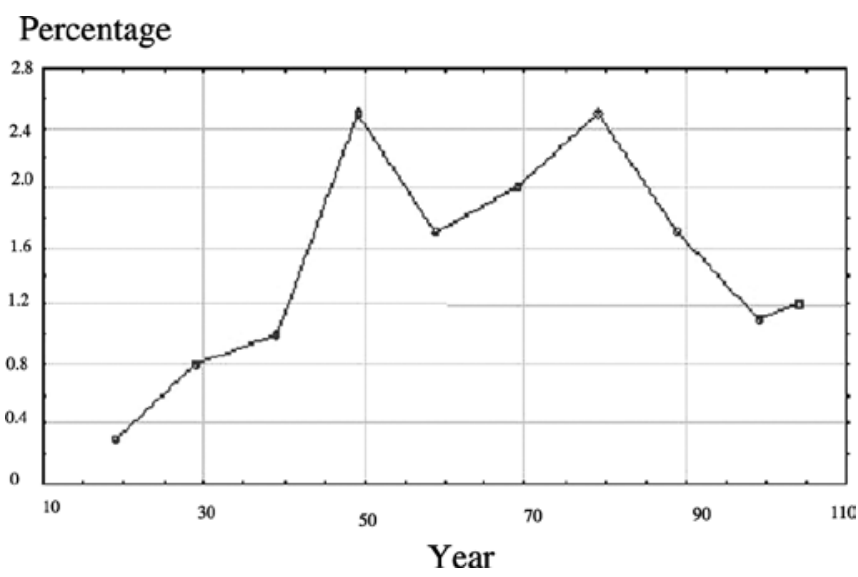

Figure 1. The change in relative incidence of stump cancers, as percentage of all invasive cervical cancers treated at Radiumhemmet (1914-2004).

Table I. Age distribution among women with stump carcinoma of the cervix.

\begin{tabular}{lccll}
\hline & No. & Min & Max & Mean \\
\hline SCC & & & & \\
$\quad$ Cases & 144 & 36 & 84 & 61.4 \\
Controls & 415 & 35 & 86.6 & 59.9 \\
AC & & & & \\
Cases & 17 & 37 & 82 & 57.9 \\
$\quad$ Controls & 36 & 36 & 83 & 57.8 \\
\hline
\end{tabular}

SCC, squamous cervical cancer, AC, adenocarcinomas.

survival corrected for intercurrent deaths was calculated for cases and controls (1). Prior to start, the study was approved by the Karolinska Institutet Ethics Board.

\section{Results}

The change in relative incidence of stump cancers during the study period is shown in Fig. 1. Mean patient age in this study was 60.1 years, range $36-84$ years. For distribution by age and histology among cases and controls, see Table I. Mean parity in the cervical stump carcinoma series was 1.6.

Reasons for subtotal hysterectomy. The predominant reasons for subtotal hysterectomy were fibromyoma and menometrorrhagia (78\%). Other reasons were noticed: inflammatory process and pain, ovarian mass, uterus rupture during the labor and unknown reasons (12\%). Mean time interval between subtotal hysterectomy and diagnosis of stump cancer was 17.6 years, range 1-46 years. For three patients the interval was 1-2 years, for 7 patients 3-5 years, and all others had a 5-year interval or more.

Histopathological findings. Of 161 stump cancer cases, 144 $(89 \%)$ were SCCs and the remaining 17 cases (11\%) were AC (Table IIA).
Table II. Histopathological and stage distributions.

A, Histopathological distribution by cases and controls

\begin{tabular}{lrrrrr}
\hline & \multicolumn{2}{c}{ Cases } & & \multicolumn{2}{c}{ Controls } \\
\cline { 2 - 3 } \cline { 5 - 6 } Histopathology & No. & & & No. & $\%$ \\
\hline SCC & 144 & 89.4 & & 415 & 91.9 \\
AC & 17 & 10.6 & & 36 & 9.2 \\
\hline
\end{tabular}

B, Stage distribution by cases and controls

\begin{tabular}{lrrrrr}
\hline & \multicolumn{2}{c}{ Cases } & & \multicolumn{2}{c}{ Controls } \\
\cline { 2 - 3 } \cline { 5 - 6 } SCC stage & No. & $\%$ & & No. & $\%$ \\
\hline I & 41 & 28.5 & 120 & 29.1 \\
II & 73 & 50.7 & 211 & 51.1 \\
III & 21 & 14.6 & 58 & 14.0 \\
IV & 7 & 4.9 & 24 & 5.8 \\
AC stage & & & & \\
I & 6 & 3.5 & 13 & 3.6 \\
II & 8 & 47.1 & 15 & 41.7 \\
III & 2 & 11.8 & 7 & 19.4 \\
IV & 1 & 5.9 & 1 & 2.8 \\
& & & & & \\
\end{tabular}

SCC, squamous cervical cancer, AC, adenocarcinomas.

Table III. Stage distribution among all cervical cancer $(n=8028)$ treated during 1959-2004.

\begin{tabular}{lr}
\hline Stage & $\%$ \\
\hline I & 38.60 \\
II & 40.75 \\
III & 12.45 \\
IV & 8.20 \\
\hline
\end{tabular}

Stage. Clinical staging was performed according to the FIGO classification for cervical cancer. Due to the long time span for the study the lowest common denominator namely the principal stage groups I, II, III, IV had to be used (1).

In our study most patients with SCC (73 cases) were classified as stage II (50.7\%). The majority of patients with AC (8 cases) were also diagnosed as stage II (47.1\%) (Table IIB). Stage distribution among all CC $(n=8028)$ is shown in Table III.

Symptoms. In $80 \%$ of cases, women were initially motivated to seek medical attention because of symptoms. Postcoital, intermenstrual, or postmenopausal bleeding was present in 88 cases. Eighteen women suffered from vaginal discharge. Fifteen women sought consultation due to pain or pain combined with vaginal bleeding or discharge. In 9 cases, the 
Kaplan \& Meier survivorship function

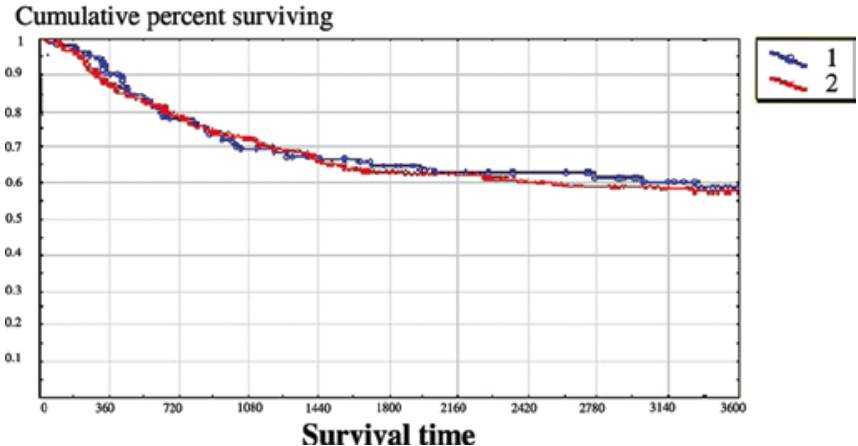

Figure 2. Cancer of the cervical stump line (1) compared with matched controls with cancer of the cervix in intact uterus line (2). Cumulative causespecific survival rate (Log-rank 0.79, Cox-Mantel 0.79).

cervix was found to be clinically abnormal during routine examination for cytology. No information was specified in $19 \%$ of the records.

Treatment. Treatment for carcinoma of the uterine stump followed mainly the Radiumhemmet individualized, intermittent, intensive treatment method for cervical cancer, two brachytherapy treatments with a three-week interval, followed by external irradiation. If possible a small radium or cesium implant was applied to the cervix, combined with a vaginal implant covering the cervical os and vaginal fornices. In a few cases during the latter part of the period, brachytherapy was followed by surgery (vaginal cervicectomy plus pelvic lymph node dissection). Some cases of recurrent cancers following completed external irradiation underwent surgery. A few recurrent cases were treated with chemotherapy. Three cases were treated exclusively with vaginal radium application, one because of poor general health, the other two because of advanced age. The radiation dose administered for stump cancer by intracavitary application was lower than that administered in comparable cases of regular CC.

Our study produced no evidence that long-term prognosis is worse for radiologically treated SCC of the uterine stump than for regular CC.

Follow-up. All cases were actively followed up at Radiumhemmet with regular examinations for at least 10 years. In some cases follow-up was carried out via correspondence with patients and their local doctors. Follow-up was continued until the end of the life and the register is complete except for a small inevitable loss due to the emigration. Death certificates were obtained from church records and in more recent years through the Swedish Cause of Death register.

Cumulative cause-specific actuarial survival rate. The cumulative cause-specific survival rate for patients treated for cervical stump cancer did not differ significantly from that of a control series matched for age, stage, and histopathology derived from the total cohort of $\mathrm{CC}$ patients treated at Radiumhemmet during the same time period (Log-rank 0.79, Cox-Mantel 0.79), Fig. 2.
Kaplan \& Meier survivorship function Cumulative percent surviving

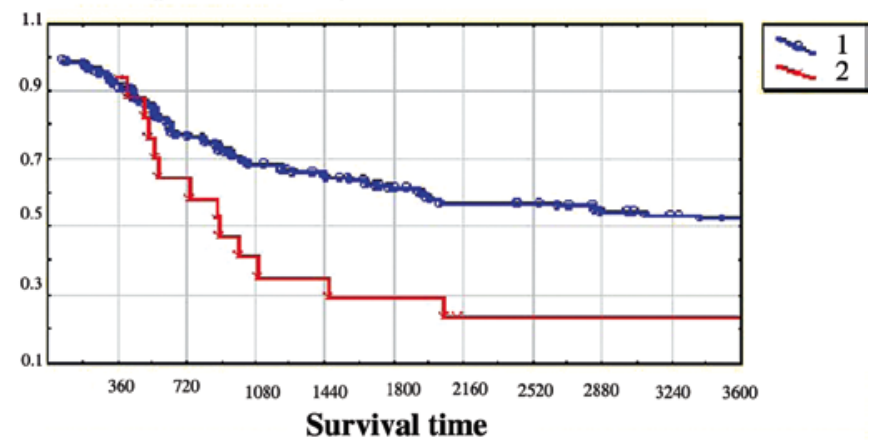

Figure 3. Carcinoma of the cervical stump. Cumulative cause-specific survival rate by histopathology. SCC (1) compared with adenocarcinomas (2). (Log-rank $\mathrm{p}=0.027$, Cox-Mantel $\mathrm{p}=0.015$, Cox F-test $\mathrm{p}=0.01$ ).

The cumulative cause-specific survival rate was significantly worse for AC than for SCC of the cervical stump (Log-rank $\mathrm{p}=0.027$, Cox-Mantel $\mathrm{p}=0.015$, Cox F-test $\mathrm{p}=0.01$ ), Fig. 3.

\section{Discussion}

Carcinoma of the cervical stump is seen in 1-3\% of patients with a history of subtotal abdominal hysterectomy (SAH) and thereby accounts for 3-9\% of all cervical carcinomas $(2,3,4)$. The changes in the relative incidence of stump cancers varying between 0.4 and $2.4 \%$ which we have observed in Radiumhemmet during the period 1914 until 2004 therefore should reflect the extent of SAH in the catchment area with a delay of about 17 years on the average $(2,3)$. Altogether with an average relative incidence of $2 \%, 360$ cases of stump cancer among $18.000 \mathrm{CC}$ at Radiumhemmet were treated between 1914-2004. Among them we have studied 161 cases diagnosed and treated during 1959-2004. We have updated the material and current arguments for and against.

The mean age for the stump cancers (SCC) was 61.4 years compared with 52.7 years for all cervical cancer cases, which may add to a poorer effect of the screening.

We have no reason to believe that women who have undergone SAH are under higher risk to develop cervical cancer than women with an intact uterus. Due to the latency period between SAH and the diagnosis of carcinoma of the cervical stump, it is likely to be a long time before changes in surgical technique influence the incidence of stump cancer. Such long intervals also render prospective studies on the effect of different treatment techniques difficult.

Furthemore, we have no evidence that the screening programs should be more efficient in detecting cancers after SAH. On the contrary, some patients and their family doctors may not have received adequate information about the extent of the hysterectomy and therefore may have erroneously concluded that the patient no longer needed cervical cancer screening (Pap smear test).

The stump cancer cases in our study show a worse stage profile compared with the cancer cases in intact uterus, which adds to the suspicion that the Pap smear test is less effective in these cases. Even if we have found that the age, stage, and 
histopathology matched controls have a similar prognosis compared with the stump cancer cases the difference in stage distribution between all cervical cancers and the stump cancers is negative for the stump cancers. Furthemore, the worse prognosis for the adenocarinoma cases compares with the squamous cases even if based on small numbers is a negative finding. The relative importance of $\mathrm{AC}$ is increasing in all hospital series.

The predominant way of removing the uterus from the 1950s was total abdominal hysterectomy even for benign condition, introduced in 1929 by the American surgeon Richardson, to avoid discharge from the cervical stump and development of the carcinoma of the cervix (5). As a rule, gynecologists have not believed the cervix to play an important physiological role. However, as early as the 1960s few studies reported in some women that the uterus was important in the physiology of the female orgasm (6) which resulted in an era advocated preserving the cervix through a more conservative approach to hysterectomy. Recent reports from hospitals in the US and Europe, with much of this research having been carried out in Scandinavia, reveal an increased number of SAH over the last two decades. In Denmark the rate increased enormously over a 10-year period in the $1990 \mathrm{~s}$ (7) by about $459 \%$ from 198 SAH to 1104 SAH. Many claims about the advantages of preserving the cervix have been made over the years.

In summary, no apparent long-term benefits of preserving the cervix have yet been discovered with respect to sexual, bladder, or bowel function.

The main advantages of subtotal hysterectomy are shorter surgery, less intraoperative blood loss, and reduction of febrile episodes. Moreover, improvements in technology have further reduced the operation time for the laparoscopic procedure (8). With trained gynecologic surgeons no main differences in time consumptions, bleedings and infections between total hysterectomies or subtotal hysterectomies would be an argument.

In conclusion, the main disadvantage is still the risk of developing cervical stump cancer and the requirement for regular cervical screening following surgery (9). Our study shows that the total effect of stump cancers following subtotal hysterectomies is not to be neglected. Although our randomized comparance did not demonstrate a worse prognosis for the stump cancers we have to take into account that the worse stage distribution in reality gives totally a poorer end result. Adenocarcinomas in the stump seems to carry a worse prognosis, even if their small numbers allow for large random error $(2,10)$. Earlier studies have shown higher complication rates following treatment (surgical as well as radiological) for stump cancer, possibly due to anatomical changes resulting from subtotal hysterectomy $(2,11-14)$.

\section{Acknowledgements}

This study was supported by grants from the Swedish Cancer Foundation (070623, CAN 2007/1044), the Cancer Society in Stockholm and the Stockholm County Council, and AFA Sweden.

\section{References}

1. Pecorelli S, Zigliani L and Odicino F: Revised FIGO staging for carcinoma of the cervix. Int J Gynaecol Obstet 105: 107-108, 2009.

2. Hellström AC, Sigurjonson T and Pettersson F: Carcinoma of the cervical stump. The radiumhemmet series 1959-1987. Treatment and prognosis. A case control study. Acta Obstet Gynecol Scand 80: 152-157, 2001.

3. Pettersson F: Secondary prevention. Screening for carcinoma of the cervix. In: Cancer in Women. Kavanagh JJ, et al (ed). Blackwell Science, Oxford, pp240-250, 1998.

4. Hannoun-Lévi JM, Peiffert D, Hoffstetter S, Luporsi E, Bey P and Pernot M: Carcinoma of the cervical stump: retrospective analysis of 77 cases. Radiother Oncol 43: 147-153, 1997.

5. Richardson EH: A simple technique for abdominal panhysterectomy. Surg Gynecol Obst 48: 248-251, 1929.

6. Kilkku P, Lehtinen V, Hirvonen T and Grönros M: Abdominal hysterectomy versus supravaginal uterine amputation: psychic factors. Ann Chir Gynaecol 76 (Suppl. 202): 62-67, 1987.

7. Gimbel H, Settnes A and Tabor A: Hysterectomy on benign indication in Denmark 1988-1998. A register based trend analysis. Acta Obstet Gynecol Scand 80: 267-272, 2001.

8. Garry R: The place of subtotal/supracervical hysterectomy in current practice. BJOG 115: 1597-1600, 2008.

9. Pricop M, Cocos M, Aursulesci D, Strat L, Gafencu C and Bendescu M: Cancer of the cervical stump. Rev Med Chir Soc Med Nat Iasi 103: 161-162, 1999.

10. Goodman HM, Niloff JM, Buttlar CA, et al: Adenocarcinoma of the cervical stump. Gynecol Oncol 35: 188-192, 1989.

11. Kottmeier HL: Surgical and radiation treatment of carcinoma of the uterine cervix. Experience by the current individualized Stockholm Technique. Acta Obstet Gynecol Scand 43 (suppl. 2): $1-48,1964$

12. Kottmeier HL and Gray MJ: Rectal and bladder injuries in relation to radiation dosage in carcinoma of the cervix. Am J Obstet Gynecol 82: 74-82, 1961.

13. Miller BE, Copeland LJ, Hamberger AD, et al: Carcinoma of the cervical stump. Gynecol Oncol 18: 100-108, 1984.

14. Barillot I, Horiot JC, Cuisenier J, et al: Carcinoma of the cervical stump: a review of 213 cases. Eur J Cancer 29: 1231-1236, 1993. 